\title{
Prevention and Treatment of Methotrexate-induced Hepatotoxicity: Potential of Natural Phytobioactive Compounds
}

Michael Adu-Frimpong ${ }^{\mathrm{a}^{*}}$, James Abugri ${ }^{\mathrm{a}}$, Bridget Osei Henewaah Annor ${ }^{\mathrm{b}}$

[*] Corresponding Author: Michael Adu-Frimpong

a Department of Applied Chemistry and Biochemistry, Faculty of Applied Sciences, CK Tedam University of Technology and Applied Sciences, Navrongo, UK-0215-5321, Ghana

${ }^{\mathrm{b}}$ Arthur Labatt Family School of Nursing, Western University, London and Ontario, Canada

Corresponding author's email: madufrimpong@cktutas.edu.gh

\begin{abstract}
Methotrexate (MTX) is a potent drug for the treatment of various diseases globally amidst being a chemotherapeutic and immunosuppressant agent. However, hepatotoxicity induced by MTX could be life-threatening if left untreated. Folate supplementation is concurrently applied to reduce the adverse effects of MTX, albeit efficacy compromise. Therefore, there is the need to understand the process for the prevention and treatment strategies for MTX induced hepatotoxicity $(\mathrm{MIH})$. In recent times, preliminary preclinical and clinical findings indicate the potential of natural phytobioactive compounds for $\mathrm{MIH}$ prevention and treatment. This mini review therefore summarizes proposed mechanisms of MIH and recent advances in the prevention and treatment prospects of natural phytobioactive compounds on MIH.
\end{abstract}

Keywords: Phytobioactive compounds; anti-inflammatory; anti-oxidant; methotrexate; hepatotoxicity; oxidative stress 


\section{Introduction}

Liver diseases are considered the most prevalent disorders worldwide and cover broad hepatic pathologies ranging from simple steatosis to chronic hepatitis, fibrosis, cirrhosis, hepatocellular carcinoma and acute liver failure [1-4]. This is worrisome as hepatic disorders generally result in disruption of the structural integrity of liver, thereby impeding vital functions in the maintenance and regulation of body homeostasis. One of such disorders causing havoc on human health is drug induced hepatotoxicity (DIH), which is defined as chemical-driven liver damage. This condition is well-documented to include some herbal medicines (administration of these agents in therapeutic windows or overdoses might damage liver) and chemicals-derived from industries and laboratories and industries and natural chemicals such as remedies of plants and microcystins might cause injury to liver. Currently, over 900 medications are culpably to be involved in inducing hepatic injury (termed as hepatoxins), which culminate in the major reason leading to drugs being unapproved or withdrawn [5-7].

Methotrexate (MTX) formerly termed as amethopterin, and a competitive dihydrofolate reductase inhibitor, has effectively been employed in the treatment of various rheumatological, oncological, dermatological, ectopically pregnancy, pancytopaenia and disorders of inflammation [8-11]. However, MTX is a well-known to induce hepatotoxicity in both humans [12] and animal models [13], albeit being the anchor drug for the treatment of rheumatoid arthritis and psoriasis [14] due to its cost effectiveness and potency. This in turn results in toxicity induced withdrawals in up to $30-50 \%$ patients, thereby limiting its use as well as replacement with more expensive and toxic therapies $[15,16]$. Putatively, the lifethreatening MTX induced hepatotoxicity (MIH) is caused by several mechanisms, nonetheless oxidative stress [17] and inflammation [18] have been well established. In view 
of this, MTX is concurrently prescribed with folic acid [15], nevertheless supplementation of folic acid and its derivatives are speculated to diminish the therapeutic efficacy of MTX, while their benefits are also controversial [19]. Due to these drawbacks, there has been an increasing search for novel strategies to reduce hepatotoxicity while optimising the efficacy of MTX [20]. Recently, supplementation of natural anti-oxidative and anti-inflammatory phytobioactive compounds has been documented to ameliorate MIH [21-23].

To the best of our knowledge no review has been documented on the potential of natural phytobioactive compounds for treatment and prevention of MIH. This mini review therefore summarizes proposed mechanisms of MIH, recent advances in the prevention and treatment prospects of natural phytobioactive compounds on $\mathrm{MIH}$, as well as suggests development of therapies involving concurrent use of natural phytoconstituents with MTX.

\section{Mechanisms of MTX-induced hepatotoxicity}

Generally, DIH is manifested as a silent sub-clinical disorder or in association with several clinical conditions $[1,24]$. For effective alleviation of MIH, the exact mechanism should be clearly understood, however this is not the case [9]. Nonetheless, recent investigations have hypothesised various mechanisms as playing vital roles in $\mathrm{MIH}$, namely anti-oxidant defences coupled with increased oxidative stress, inhibition and activation of nuclear factorerythroid-2 related factor 2-(Nrf2) anti-oxidant defence response (ARE)-nuclear factor kappa B (Nrf2-ARE-NF-kB) crosstalk, down-regulation of PPAR- $\gamma$ as well as release of proinflammatory and apoptotic mediators [9,13,14,25,26]. Notwithstanding these results, understanding the detailed mechanism underlying MIH could help in attenuating the adverse hepatotoxic side effects of amethopterin therapy. 


\subsection{Oxidative stress, lipid peroxidation and MTX-induced hepatotoxicity}

Several lines of evidences have documented the association between incidence of MIH and oxidative stress. These studies [14,27] suggest that MTX injured liver by negatively modulating mitochondrial machinery, wherein it resulted in uncontrolled production of reactive oxygen species (ROS) [14,28]. Consequently, this process leads to disruption of cellular macromolecules coupled with initiation of lipid peroxidation cascade and its concomitant cell death [29]. Additionally, it was established about two decades ago that MTX concentration is prolonged in intracellular via its conversion to polyglutamates in the liver.

Accordingly, this contributes to hepatotoxicity through cellular NADPH unavailability and glutathione reductase inhibition [30]. To date, this hypothesis has neither been corroborated nor refuted, thus further studies are needed for current understanding of this mechanism.

The MIH has been implicated in the depletion of mitochondrial enzymatic and non-enzymatic anti-oxidant defence systems through overproduction of ROS [31-33]. In recent times, emerging evidence demonstrates the involvement of Nrf2 Kelch-like erythroid cell-derived protein-1 (Keap-1) ARE-(Nrf2-Keap1-ARE) signalling pathway in regulating cellular resistance to oxidants [23,29]. As a class of basic-region leucine zipper (bZIP) protein, Nrf2 is well established to protect tissues against oxidative-induced injury via increased expression of cellular anti-oxidant defence proteins. Upon inducement of uncontrolled ROS production by MTX, the Nrf2 (generally suppressed in cytoplasm by Keap-1) [34] is activated. After transport of Nrf2 to nucleus, it binds to the ARE, which subsequently initiates transcription of anti-oxidative genes such as haem oxygenase 1 (HO-1) and NADPH quinone oxidoreductase-1 (NQO-1) [35]. Mechanistically, Nrf2 is activated via induction in two fronts, which are suppression and activation of Nrf2 under basal condition by inducers [36]. In this 
regard, there is an unmet need to identify natural phytobioactive compounds that can boost Nrf2 activation. However, with the emerging concept of Nrf2 functioning in double-edge sword manner [37,38], further investigations are required to unearth the exact impact of Nrf2 activation as well as actual role of its suppression in MIH.

Oxidative stress-induced lipid peroxidation is among the numerous mechanisms through which MTX causes liver injury. The uncontrolled ROS generated through MTX metabolism in liver attacks hepatocellular membranes resulting in lipid peroxidation, which potentially forms toxic lipid-derived aldehydes (LDAs), viz., acrolein, 4-hydroxy-2-nonenal (HNE) and malondialdehyde (MDA) [38]. In accordance with this hypothesis, several authors have shown that MTX administration in rat models may induce evidential elevations of MDA and nitric oxide (NO) with concomitant decrease in the activities of catalase, glutathione (GSH) and superoxide dismutase (SOD) [9,18,39].

Despite the highly reactive NO culpability in the pathological process of hepatotoxicity induced by MTX [40], other authors [41] have posited that NO possesses hepatoprotective effect. Thus, NO acts a double-edged sword by reacting with superoxide radical, forming potent lipid peroxidation-inducing agent, peroxynitrite, while protecting the liver through inhibition of tumour necrosis factor-alpha (TNF- $\alpha$ ) via NF-kB subunits modifications [41].

\subsection{Role of inflammatory and apoptotic factors in MTX-induced hepatotoxicity}

The involvement of inflammatory processes in hepatotoxicity induced by chemicals has been reported to occur through production of mediators that can cause liver damage or impede repair [42]. The release of inflammatory mediators during hepatic injury have been documented to include interleukin-1 $\beta$ (IL-1 $\beta$ ), IL-6, IL-8, NO and TNF- $\alpha[40,43,44]$ in animal models. 
These cytokines and inflammatory mediators in concert with "master regulator of the inflammatory response (TNF- $\alpha$ ) are capable of directly injuring liver [45]. Moreover, it has been established that inflammation is linked to apoptosis through TNF- $\alpha$, which plays vital role in the homeostasis of liver [46]. Existing literature suggests that increased TNF- $\alpha$ expression result in the activation of apoptotic pathways (anti-apoptosis-NF- $\kappa \mathrm{B}$ and proapoptosis-caspases), [47] which in turn cause MIH [43].

Further, it is speculated that proteins such as cyclooxygenases- 2 and inducible NO synthase (iNOS, both regulated by NF- $\kappa B$ ) generally influences the biological effect of TNF- $\alpha$ [48]. Also, it is possible MTX induced NF- $\kappa \mathrm{B}$ signalling activation via 5-aminoimidazole-4carboxamideribonucleoside (AICAR) [49] since its active and storage form, methotrexate polyglutamates (MTXGlu) can cause intracellular accumulation of AICAR through the inhibition of AICAR transformylase [50], thereby resulting in inflammation.

Moreover, TNF- $\alpha$ receptor-1 (TNFR-1) activation is assumed to be harbinger, wherein it is associated with cellular apoptosis initiation via activation of different types of caspases [51] (Figure 1). Among this family of protease enzymes is caspase-3, which often activates protease for cell death, thereby catalysing particular splitting of various important proteins in cells which consequently culminates in MTX-induced apoptosis [52]. Judging from preclinical studies, it seems plausible that inflammation- and stress-related signalling pathways are the underlying mechanism of MIH. Thus, therapeutic strategies aimed at attenuating oxidative stress and its concomitant inflammation as well as enhancing cellular anti-oxidants can be explored to potentially prevent and treat $\mathrm{MIH}$. 


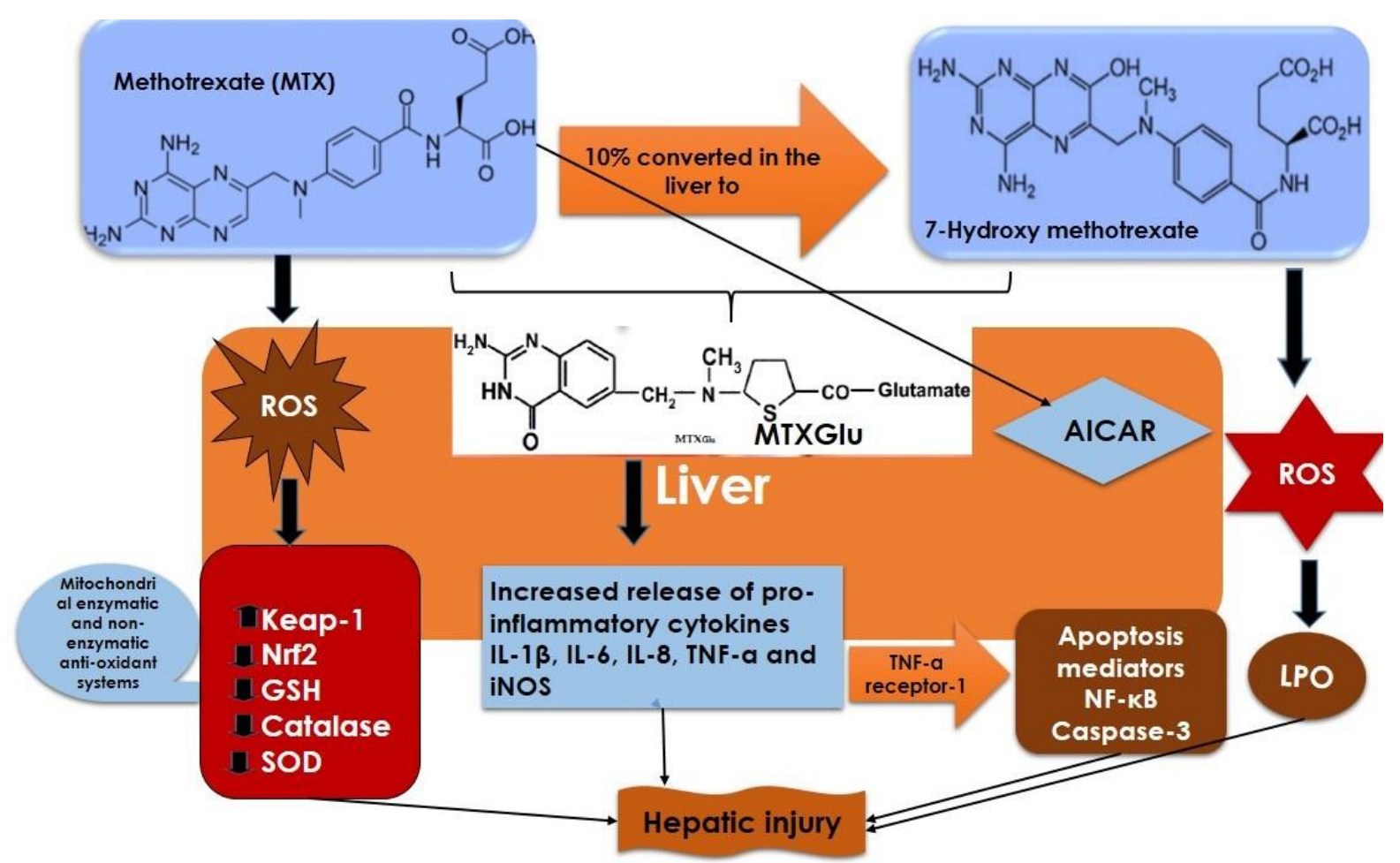

Figure 1. A schematic representation of possible mechanism underlying hepatotoxicity induced by MTX through oxidative stress-related inflammation, lipid deterioration by ROS and apoptosis. AICAR; 5-aminoimidazole-4carboxamide-ribonucleoside, GSH; glutathione, Keap-1: Kelch-like erythroid cell-derived protein-1, IL-1 $\beta$, IL-6 and IL-8; Interleukin-1 $\beta,-6$ and -8, iNOS; inducible nitric oxide synthase, LPO; Lipid peroxidation, MTXGlu; Methotrexate polyglutamates, NF-kB; nuclear factor kappa B, Nrf2; nuclear factor-erythroid2 related factor 2, ROS; reactive oxygen species, SOD; superoxide dismutase, TNF- $\alpha$; tumour necrosis factor alpha

\section{Prevention and treatment prospects of natural phytobioactive compounds}

Long-term use of MTX as first line treatment for chronic diseases such as rheumatoid arthritis and cancer normally result in increase in aminotransferases (also known as transaminitis) [53], which has recently received much recognition in clinical research [39]. Currently, prevention and treatment options for the aforementioned diseases are through concomitant use of folate supplementation (folic acid or folinic acid) and MTX to specifically 
minimize adverse effects including hepatotoxicity. Although, some studies have hinted that folate co-administration may not compromise MTX efficacy [54] but results of a post hoc analysis of two randomised control investigations suggested otherwise [55]. It is possible $\mathrm{MIH}$ is unrelated to folate antagonism unlike the other adverse effects such as anaemia, neutropaenia, stomatitis and oral ulcers [56]. Therefore, there is urgent need to search for novel preventive and treatment strategies. Current evidences indicate that supplementation of natural phytobioactive compounds with anti-oxidants properties might protect liver against MIH [25,27,33,52]. However, these preliminary findings should be confirmed in further studies through randomised clinical trials.

Natural products and its derived active constituents normally known as phytobioactive compounds have recently been explored in the treatment of several diseases [57-60]. In recent times, our exploits in phytocompounds research support the assertion that natural products have the potential to prevent and treat oxidative stress-related inflammation which underlie liver disorders [61,62]. Exemplary, Zhang and his colleagues established previously that natural bioactive phytocompounds from fruits and vegetables could ameliorate the incidence of several illnesses, while those possessing anti-oxidant properties could potentially reduce severe adverse effects of anti-tumour drugs in Chinese women (Zhang et al., 2009) in particular and global population as a whole. Invariably, there is growing optimism that these natural phytocompounds when supplemented with MTX could ameliorate its associated hepatotoxicity.

The prevention and treatment prospect of natural phytobioactive compounds on MIH for the past five years is summarized in Table 1. The preclinical and clinical evidences that we reviewed suggest that natural phytobioactive compounds were mainly comprised of polyphenols, saponins, isoquinoline alkaloids, flavonoid and phenols, which generally 
exhibited anti-oxidant, anti-inflammatory and anti-hepatotoxic properties against MIH (Table 1). Thus, the authors suggested that the understudied natural phytobioactive compounds exhibited promising hepatoprotective potential against hepatotoxicity of MTX. This in turn results in the maintenance of enzyme homeostasis in liver while providing increased antioxidant defence against oxidative-induced free radicals [63]. This is not surprising as several natural phytocompounds with anti-oxidant activities have been documented to protect liver against various hepatoxicans $[6,22,64]$. As postulated earlier by Brewer, the potency of wide range of anti-oxidant agents is proportional to the presence of hydroxyl $(\mathrm{OH})$ groups on their aromatic ring(s) [65]. This implies that phytocompounds with higher number of $\mathrm{OH}$ groups are likely to exert more effective anti-oxidant properties.

Besides, natural phytocompounds demonstrate better anti-oxidative effect based on the diversity of their chemical structures and bioactivities compared to the currently available synthetic ones in the commercial functional foods and nutraceuticals [66]. In this regard the search for novel natural phytonutrients with anti-oxidant effect still remains a burgeoning field.

Due to growing body of evidences which suggest the overproduction of ROS and its associated oxidative stress-related inflammatory responses in playing crucial roles in the pathogenesis of various disorders [67], natural agents with both anti-inflammatory and antioxidant properties could be explored further to affirm their potency against MIH. 
Table 1: Prevention and treatment prospect of natural phytobioactive compounds on MTX induced hepatotoxicity (MIH)

\begin{tabular}{llll}
\hline & Research & \\
& Bioactivity & type & \\
& & \\
\hline
\end{tabular}

Hibiscus sabdariffa, Anti-oxidant, anti-

Solanum melongena, inflammatory, anti-

Chlorogenic acid

Prunus persica and Prunus apoptotic and anti-

domestica

hepatotoxic

Thymoquinone Nigella sativa Oil

Anti-oxidant,

Clinical

antihepatotoxic

Anti-oxidant, anti-

Lauric acid

Virgin coconut oil (Cocos hepatotoxic and nucifera)

anti-lipid

Preclinical [25]

peroxidation

$18-\beta-$

Anti-oxidant, anti-

glycyrrhetinic

Licorice root extract inflammatory, anti-

acid

(Glycyrrhiza glabra) hepatotoxic and

Preclinical [14]

anti-apoptotic

Anti-oxidant, anti-

inflammatory, anti-

Berberine Coptis chinensis hepatotoxic, anti-Preclinical [9]

apoptotic and anti-

lipid peroxidation

Berberine Coptidis rhizome Anti-oxidant, anti- Preclinical [68] 


$\begin{array}{lc}\text { (Rhizoma coptidis) } & \text { hepatotoxic and } \\ & \text { anti-lipid } \\ & \text { peroxidation } \\ & \text { Anti-oxidant, anti- } \\ & \text { inflammatory, anti- }\end{array}$

Ellagitannins (as

punicalagins and hepatotoxic, anti- Preclinical [23]

(Punica granatum L.)

free ellagic acid) apoptotic and anti-

lipid peroxidation

Anti-oxidant, anti-

Resveratrol

Grapes, blueberries, hepatotoxic and

raspberries, mulberries anti-lipid

Preclinical [21]

peroxidation

Turmeric

Curcuma longa L.,

Anti-oxidant, anti-

Zingiberaceae

inflammatory and Preclinical [33]

anti-hepatotoxic

Anti-oxidant, anti-

Green tea, gall nut, grapes,

hepatotoxic and

Gallic acid red wine, hops, oak bark

anti-lipid

etc.

peroxidation

\section{Conclusions and future direction}

Up to now, there is no approved treatment strategy designed to cure MIH. Normally, folate is concurrently administered with MTX; however this approach has yielded inconclusive results. Current evidence hypothesises that MIH is initiated and progressed through oxidative 
stress-related inflammation, peroxidation and apoptosis. However, the process by which the metabolism of MTX induced overproduction of ROS is not clearly understood. Therefore, understanding the mechanisms of underlying MTX hepatotoxic effect would unearth the type of treatment strategies capable of preventing and treating MIH in humans. In view of this, pharmacological interventions of choice should be aimed at alleviating all the underlying mechanisms of MIH.

Preliminary preclinical and clinical findings show that natural phytobioactive compounds could prevent and treat MIH. This is promising, albeit several clinical trials needed to evaluate the actual effectiveness of this treatment option. In subsequent investigations, scientists should explore concurrent use of these natural phytonutrients with MTX and further assess their effects on MTX efficacy. Moreover, through nanotechnology techniques coencapsulation of MTX and natural phytocompounds can be explored for the prevention and treatment of MIH.

\section{Acknowledgements}

Our sincere thanks go to authors whose published articles were used in this review

\section{References}

[1] W. Larson, AM, Polson, J, Fontana, RJ, Davern, TJ, Lalani, E, Hynan, LS, Reisch, JS, Schiodt, FV, G. Ostapowicz, G, Shakil, AO and Lee, Acetaminophen-induced acute liver failure: results of a United States multicenter, prospective study, Hepatology. 42 (2005) 1364-1372.

[2] A. Loguercio, C and Federico, Oxidative stress in viral and alcoholic hepatitis, Free Radic Biol Med. 34 (2003) 1-10. 
[3] C. Lieber, Alcoholic fatty liver: its pathogenesis and mechanism of progression to inflammation and fibrosis, Alcohol. 34 (2004) 9-19.

[4] N. Yuan, L and Kaplowitz, Mechanisms of drug-induced liver injury, Clin. Liver Dis. 17 (2013) 507-518.

[5] L. Kaplowitz, N, Win, S, Than, TA, Liu, ZX and Dara, Targeting signal transduction pathways which regulate necrosis in acetaminophen hepatotoxicity, J Hepatol. 63 (2015) 5-7.

[6] A. Mahmoud, Hesperidin protects against cyclophosphamide-induced hepatotoxicity by upregulation of PPARg and abrogation of oxidative stress and inflammation, Can. J. Physiol. Pharmacol. 92 (2014) 717-724.

[7] P. Pandit, A, Sachdeva, T and Bafna, Drug-Induced Hepatotoxicity: A Review, J. Appl. Pharm. Sci. 2 (2012) 233-243. https://doi.org/10.7324/JAPS.2012.2541.

[8] M. Ayad, MW, El Naggar, AA, and El Naggar, MTHFR C677T polymorphism: association with lymphoid neoplasm and effect on methotrexate therapy, Eur. J. Haematol. 93 (2014) 63-69. https://doi.org/10.1016/j.biopha.2017.07.101.

[9] S. Mahmoud, AM, Hozayen, WG and Ramadan, Berberine ameliorates methotrexateinduced liver injury by activating Nrf2/HO-1 pathway and PPAR-gamma, and suppressing oxidative stress and apoptosis in rats, Biomed. Pharmacother. 94 (2017) $280-291$.

[10] E. Sakthiswary, R and Suresh, Methotrexate in systemic lupus erythematosus: a systematic review of its efficacy, Lupus, Lupus. 23 (2014) 225-235.

[11] W. Amissah-Arthur, M-B, Baah, Methotrexate-induced pancytopenia and mucositis caused by medication error, Ghana Med J. 54 (2020) 68-71. 
[12] E. Hagag, AA, Elaal, AMA, Elsheik, A and Elzamarany, Protective Effect of Nigella sativa Oil against Methotrexate Induced Hepatotoxicity in Children with Acute Lymphoblastic Leukemia, J Leuk. 1 (2013) 1-7.

[13] S. Ali, N, Rashid, S, Nafees, S, Hasan, SK, Shahid, A, Majed, F and Sultana, Protective effect of Chlorogenic acid against methotrexate induced oxidative stress, inflammation and apoptosis in rat liver: An experimental approach, Chem. Biol. Interact. 272 (2017) 80-91. https://doi.org/10.1016/j.cbi.2017.05.002.

[14] W. and Mahmoud, AM, Hussein, OE, Hozayen, S. El-Twab, Methotrexate hepatotoxicity is associated with oxidative stress, and down-regulation of PPARgamma and Nrf2: Protective effect of 18b-Glycyrrhetinic acid, Chem. Biol. Interact. 270 (2017) 59-72.

[15] S. Dhir, V, Sandhu, A, Kaur, J, Pinto, B, Kumar, P, Kaur, P, Gupta, N, Sood, A, Sharma, A and Sharma, Comparison of two different folic acid doses with methotrexate - a randomized controlled trial (FOLVARI Study), Arthritis Res. Ther. 17 (2015) 156-165.

[16] G. Rau, R and Herborn, Benefit and risk of methotrexate treatment in rheumatoid arthritis, Clin Exp Rheumatol. 22 (2004) S83-94.

[17] H. Tousson, E, Zaki, ZT, Abu-Shaeirf, WA and Hassan, Methotrexate-induced hepatic and renal toxicity: role of L-carnitine in treatment, Biomed. Biotechnol. 4 (2014) 8592.

[18] E. Hafez, HM, Ibrahim, MA, Ibrahim, SA, Amin, A. Goma, W and Abdelrahman, Potential protective effect of etanercept and aminoguanidine in methotrexate-induced hepatotoxicity and nephrotoxicity in rats, Eur. J. Pharmacol. 768 (2015) 1-12. 
https://doi.org/10.1016/j.ejphar.2015.08.047.

[19] D. Smolen, JS1, Landewé, R, Breedveld, FC, Buch, M, Burmester, G, Dougados, M, Emery, P, Gaujoux-Viala, C, Gossec, L, Nam, J, Ramiro, S, Winthrop, K, de Wit, M, Aletaha, D, Betteridge, N, Bijlsma, JW, Boers, M, Buttgereit, F, Combe, B, Cutolo, M, Damjanov, N, EULAR recommendations for the management of rheumatoid arthritis with synthetic and biological disease-modifying antirheumatic drugs: 2013 update, Ann Rheum Dis. 73 (2014) 492-509. https://doi.org/10.1136/annrheumdis-2013204573.

[20] R. Cipriani, P, Ruscitti, P, Carubbi, F, Liakouli, V and Giacomelli, Methotrexate in rheumatoid arthritis: optimizing therapy among different formulations. Current and emerging paradigms, Clin Ther. 36 (2014) 427-35.

[21] S. Dalaklioglu, S, Genc, GE, Aksoy, NH, Akcit, F and Gumuslu, Resveratrol ameliorates methotrexate-induced hepatotoxicity in rats via inhibition of lipid peroxidation, Hum. Exp. Toxicol. 32 (2013) 662-671.

[22] M. De, S, Sen, T and Chatterjee, Reduction of oxidative stress by an ethanolic extract of leaves of Piper betle (Paan) Linn. decreased methotrexate-induced toxicity, Mol. Cell Biochem. 409 (2015) 191-197.

[23] S. Mukherjee, S, Ghosh, S, Choudhury, S, Adhikary, A, Manna, K, Dey, S, Sa, G, Das, $\mathrm{T}$ and Chattopadhyay, Pomegranate reverses methotrexate-induced oxidative stress and apoptosis in hepatocytes by modulating Nrf2-NF-кB pathways, J. Nutr. Biochem. 24 (2013) 2040-2050. https://doi.org/10.1016/j.jnutbio.2013.07.005.

[24] N. Motamedi, NDL and Kaplowitz, Clinical considerations of drug-induced hepatotoxicity, in: Compr. Toxicol., C. McQueen, Elsevier B.V., Amsterdam, The 
Netherlands, 2017.

[25] G. Famurewa, AC, Ufebe, OG, Egedigwe, CA, Nwankwo, OE and Obaje, Virgin coconut oil supplementation attenuates acute chemotherapy hepatotoxicity induced by anticancer drug methotrexate via inhibition of oxidative stress in rats, Biomed. Pharmacother. 87 (2017) 437-442. https://doi.org/10.1016/j.biopha.2016.12.123.

[26] A. Kelleni, MT, Ibrahim, SA and Abdelrahman, Effect of captopril and telmisartan on methotrexate-induced hepatotoxicity in rats: impact of oxidative stress, inflammation and apoptosis, Toxicol. Mech. Methods. $26 \quad$ (2016) 1-7. https://doi.org/10.1080/15376516.2016.1191576.

[27] S. Ali, N, Rashid, S, Nafees, S, Hasan, SK and Sultana, Beneficial effects of chrysin against methotrexate-induced hepatotoxicity via attenuation of oxidative stress and apoptosis, Mol. Cell. Biochem. 385 (2014) 215-223.

[28] P. Kolli, VK, Natarajan, K, Isaac, B, Selvakumar, D and Abraham, Mitochondrial dysfunction and respiratory chain defects in a rodent model of methotrexate-induced enteritis, Hum. Exp. Toxicol. 33 (2014) 1051-1065.

[29] V. Naik, SK and Panda, Antioxidant and hepatoprotective effects of Ginkgo biloba phytosomes in carbon tetrachloride-induced liver injury in rodents, Liver Int. 27 (2007) 393-399.

[30] G. Jahovic, N, Cevik, H, Sehirli, AO, Yegen, BC and Sener, Melatonin prevents methotrexate-induced hepatorenal oxidative injury in rats, J. Pineal Res. 34 (2003) 282-287.

[31] A. and Al Maruf, A, O’Brien, PJ, Naserzadeh, P, Fathian, R, Salimi, J. Pourahmad, Methotrexate induced mitochondrial injury and cytochrome c release in rat liver 
hepatocytes, Drug Chem. Toxicol. $41 \quad$ (2017) 1-11. https://doi.org/10.1080/01480545.2017.1289221.

[32] M. de Almeida, VG, Alves, CC, Moraes, DG, Glorimar, R and Bello, Effects of the intake of sesame seeds (Sesamum indicum L.) and derivatives on oxidative stress: a systematic review, J. Med. Food. 19 (2016) 337-345.

[33] S. Moghadam, AR, Tutunchi, S, Namvaran-Abbas-Abad, A, Yazdi, M, Bonyadi, F, Mohajeri, D, Mazani, M, Marzban, H, Kos, MJ and Ghavami, Pre-administration of turmeric prevents methotrexate-induced liver toxicity and oxidative stress, BMC Complement. Altern. Med. 15 (2015) 246-258. https://doi.org/10.1186/s12906-0150773-6.

[34] M. Kang, MI, Kobayashi, A, Wakabayashi, N, Kim, SG and Yamamoto, Scaffolding of Keap1 to the actin cytoskeleton controls the function of Nrf2 as key regulator of cytoprotective phase 2 genes, in: Proc. Natl. Acad. Sci. USA, 2004: pp. 2046-2051.

[35] A. Jaiswal, Nrf2 signaling in coordinated activation of antioxidant gene expression, Free Radic. Biol. Med. 36 (2004) 1199-1207.

[36] Q. Ma, Role of Nrf2 in oxidative stress and toxicity, Annu. Rev. Pharmacol. Toxicol. 53 (2013) 401-426. https://doi.org/10.1146/annurev-pharmtox-011112-140320.

[37] X. Ma, Q and He, Molecular basis of electrophilic and oxidative defense: promises and perils of Nrf2, Pharmacol Rev. 64 (2012) 1055-1081.

[38] M. Taguchi, K, Motohashi, H and Yamamoto, Molecular mechanisms of Keap1-Nrf2 pathway in stress response and cancer evolution, Genes Cells. 16 (2011) 123-140.

[39] E. and Akbulut, S, Elbe, H, Cengiz Eris, C, Dogan, Z, Toprak, G, Otan, E, Erdemli, T. Yusuf, Cytoprotective effects of amifostine, ascorbic acid and $\mathrm{N}$-acetylcysteine against 
methotrexate-induced hepatotoxicity in rats, World J Gastroenterol. 20 (2014) 1015810165. https://doi.org/10.3748/wjg.v20.i29.10158.

[40] R. Leitão, RF, Brito, GA, Oria, RB, Braga-Neto, MB, Bellaguarda, EA, Silva, JV, Gomes, AS, Lima-Junior, RC, Siqueira, FJ, Freire, RS, Vale, ML and Ribeiro, Role of inducible nitric oxide synthase pathway on methotrexate-induced intestinal mucositis in rodents, BMC Gastroenterol. 16 (2011) 11-90.

[41] D. Morio, LA, Chiu, H, Sprowles, KA, Zhou, P, Heck, DE, Gordon, MK and Laskin, Distinct Roles of Tumor Necrosis Factor-a and Nitric Oxide in Acute Liver Injury Induced by Carbon Tetrachloride in Mice, Toxicol. Appl. Pharmacol. 172 (2001) 4451. https://doi.org/10.1006/taap.2000.9133,.

[42] B. Luster, MI, Simeonovaa, PP, Gallucci, RM, Bruccoleri, A, Blazka, ME and Yucesoy, Role of inflammation in chemical-induced hepatotoxicity, Toxicol. Lett. 120 (2001) 317-21. https://doi.org/10.1016/S0378-4274(01)00284-3.

[43] S. Cetiner, M, Sener, G, Sehirli, AO, Eksioglu-Demiralp, E, Ercan, F, Sirvanci, B. Gedik, N, Akpulat, S, Tecimer, T and Yegen, Taurine protects against methotrexateinduced toxicity and inhibits leukocyte death, Toxicol. Appl. Pharmacol. 209 (2005) $39-50$.

[44] S. Di Paola, R, Mazzon, E, Muià, C, Crisafulli, C, Terrana, D, Greco, S, Britti, D, Santori, D, Oteri, G, Cordasco, G and Cuzzocrea, Effects of etanercept, a tumour necrosis factor-alpha antagonist, in an experimental model of periodontitis in rats, Br.J.Pharmacol. 150 (2007) 286-297.

[45] R.A. Tukov, F.F., Luyendyk, J.P., Ganey, P.E., Roth, The role of tumor necrosis factor alpha in lipopolysaccharide/ranitidine-induced inflammatory liver injury, Toxicol.Sci. 
100 (2007) 267-280.

[46] C. Tacke, F, Luedde, T and Trautwein, Inflammatory Pathways in Liver Homeostasis and Liver Injury, Clin. Rev Allerg Immunol. 36 (2009) 4-12.

[47] C. Liedtke, C and Trautwein, The role of TNF and Fas dependent signaling in animal models of inflammatory liver injury and liver cancer, Eur. J. Cell Biol. 91 (2012) 582589.

[48] J. Aggarwal, BB, Gupta, SC snd Kim, Historical perspectives on tumor necrosis factor and its superfamily:25 years later a golden journey, Blood. 119 (2012) 651-665.

[49] I. Jung, JE, Lee, J, Ha, J, Kim, SS, Cho, YH, Baik, HH and Kang, 5-Aminoimidazole4-carboxamide-ribonucleoside enhances oxidative stress-induced apoptosis through activation of nuclear factor- $\kappa \mathrm{B}$ in mouse Neuro $2 \mathrm{a}$ neuroblastoma cells, Neurosci. Lett. 354 (2004) 197-200. https://doi.org/10.1016/j.neulet.2003.10.012.

[50] J. Tian, T, Wang, Z, Zhang, Pathomechanisms of Oxidative Stress in Inflammatory Bowel Disease and Potential Antioxidant Therapies, Oxid. Med. Cell. Longev. 2017 (2017) 1-18.

[51] C. and Cubero, FJ, Singh, A, Borkham-Kamphorst, E, Nvzorova, YA, AlMasaoudi, M, Hass, U, Boekschoten, MV, Gassler, N, Weiskirchen, R, Muller, M, Liedtke, C. Trautwein, TNFR1 determines progression of chronic liver injury in the IKK $\gamma / \mathrm{Nemo}$ genetic model, Cell Death Differ. 20 (2013) 1580-1592.

[52] C.-F. Chang, C-J, Lin, J-F, Chang, H-H, Lee, G-A and Hung, Lutein Protects against Methotrexate-Induced and Reactive Oxygen Species-Mediated Apoptotic Cell Injury of IEC-6 Cells, PLoS One. $8 \quad$ (2013) 72553. https://doi.org/10.1371/journal.pone.0072553. 
[53] S. Sotoudehmanesh, R, Anvari, B, Akhlaghi, M, Shahraeeni, S and Kolahdoozan, Methotrexate Hepatotoxicity in Patients with Rheumatoid Arthritis, Middle East J. Dig. Dis. 2 (2010) 104-109.

[54] R. Whittle, SL and Hughes, Folate supplementation and methotrexate treatment in rheumatoid arthritis: a review, Rheumatology. 43 (2004) 267-271.

[55] S. Baggott, JE and Morgan, Folic acid supplements are good (not bad) for rheumatoid arthritis patients treated with low-dose methotrexate (letter), Am. J. Clin. Nutr. 88 (2008) 479-480.

[56] B. Tian, $\mathrm{H}$ and Cronstein, Understanding the Mechanisms of Action of Methotrexate: Implications for the Treatment of Rheumatoid Arthritis, Ulletin NYU Hosp. Jt. Dis. 65 (2007) 168-73. https://doi.org/10.1007/s12016-008-8091-0.

[57] J. Adu-Frimpong, M, Firempong, CK, Omari-Siaw, E, Wang, Q, Mukhtar, YM, Deng, WW, Yu, QT, Xu, X, Yu, Preparation, optimization, and pharmacokinetic study of Acid, nanoliposomes loaded with triacylglycerol-bound punicic Activity, for increased antihepatotoxic, Drug Dev Res. 79 (2018) 1-16.

[58] X. Feng, S, Yingying, Z, Firempong, CK and Xu, Preparation, characterization and pharmacokinetic studies of linalool-loaded nanostructured lipid carriers, Pharm. Biol. 54 (2016) 2320-2328. https://doi.org/10.3109/13880209.2016.1155630.

[59] X. Wang, Q, Wei, Q, Yang, Q, Cao, X, Li, Q, Shi, F, Tong, SS, Feng, C, Yu, Q, Yu, J and $\mathrm{Xu}, \mathrm{A}$ novel formulation of [6]-gingerol: Proliposomes with enhanced oral bioavailability and antitumor effect, Int. J. Pharm. 535 (2018) 308-315.

[60] Y.J. Adu-Frimpong M, Quiyu W, Firempong CK, Mukhtar YM, Yang Q, Omari-Siaw E, Lijun Z, Xu X, Novel cuminaldehyde self-emulsified nanoemulsion for enhanced 
antihepatotoxicity in carbon tetrachloride-treated mice, J Pharm Pharmacol. 71 (2019) $1324-1338$.

[61] J. Omari-Siaw, E, Wang, Q, Sun, C, Gu, Z, Zhu, Y, Cao, X, Firempong, CK, Agyare, $\mathrm{R}, \mathrm{Xu}, \mathrm{X}$ and $\mathrm{Yu}$, Tissue distribution and enhanced in vivo anti-hyperlipidemic antioxidant effects of perillaldehyde-loaded liposomal nanoformulation against Poloxamer 407-induced hyperlipidemia, Int. J. Pharm. 513 (2016) 68-77.

[62] X.X. Sun C, Li W, Liu Y, Deng W, Adu-Frimpong M, Zhang H, Wang Q, Yu J, No TitleIn vitro/in vivo hepatoprotective properties of 1-O-(4-hydroxymethylphenyl)- $\alpha$-Lrhamnopyranoside from Moringa oleifera seeds against carbon tetrachloride-induced hepatic injury, Food Chem Toxicol. 131 (2019) 110531.

[63] Z. Hussain, F, Malik, A, Ayyaz, U, Shafique, H, Rana, Z, Hussain, Efficient hepatoprotective activity of cranberry extract against CCl4-induced hepatotoxicity in Wistar albino rat model: Down-regulation of liver enzymes and strong antioxidant activity, Asian Pac. J. Trop. Med. 10 (2017) 1054-1058.

[64] S. Chen, X, Zhang, Y, Zhu, Z, Liu, H, Guo, H, Xiong, C, Xie, K, Zhang, X and Su, Protective effect of berberine on doxorubicininduced acute hepatorenal toxicity in rats, Mol. Med. Rep. 13 (2016) 3953-3960.

[65] M. Brewer, Natural Antioxidants: Sources, Compounds, Mechanisms of Action, and Potential Applications, Compr. Rev. Food Sci. Food Saf. 10 (2011) 221-247.

[66] M. White, PAS, Oliveira, RCM, Oliveira, AP, Serafini, MS, Araújo, AAS, Daniel P. Gelain, DP, Moreira, JCF, Almeida, JRGS, Quintans, JSS, Quintans-Junior, LJ and Santos, Antioxidant Activity and Mechanisms of Action of Natural Compounds Isolated from Lichens: A Systematic Review, Molecules. 19 (2014) 14496-14527. 
https://doi.org/10.3390/molecules190914496.

[67] X. Jia, Z, Babu, PVA, Chen, W and Sun, Natural Products Targeting on Oxidative Stress and Inflammation: Mechanisms, Therapies, and Safety Assessment, Oxid. Med. Cell. Longev. 2018 (2018) 1-3. https://doi.org/10.1155/2018/6576093.

[68] H. and Mehrzadi, S, Fatemi, I, Esmaeilizadeh, M, Ghaznavi, H, Kalantar, M. Goudarzi, Hepatoprotective effect of berberine against methotrexate induced liver toxicity in rats, Biomed. Pharmacother. 97 (2018) 233-239.

[69] O. Olayinka, ET Ore, A, Adeyemo, OA and Ola, Ameliorative effect of gallic acid on methotrexate-induced hepatotoxicity and nephrotoxicity in rat, J. Xenobiotics. 1 (2016) 1-21. https://doi.org/10.4081/xeno.2016.6092. 